\title{
Effective Solution of Ill-Posed Inverse Problems with Stabilized Forward Solver
}

\author{
Marcin Łoś(D), Robert Schaefer(D), and Maciej Smołka $\left.{ }^{(}\right)_{(\mathbb{D}}$ \\ Institute of Computer Science, AGH University of Science and Technology, \\ Kraków, Poland \\ \{los, schaefer, smolka\} @agh.edu.pl \\ http://www.informatyka.agh.edu.pl/
}

\begin{abstract}
We consider inverse parametric problems for elliptic variational PDEs. They are solved through the minimization of misfit functionals. Main difficulties encountered consist in the misfit multimodality and insensitivity as well as in the weak conditioning of the direct (forward) problem, that therefore requires stabilization. A complex multi-population memetic strategy $h p$-HMS combined with the PetrovGalerkin method stabilized by the Demkowicz operator is proposed to overcome obstacles mentioned above. This paper delivers the theoretical motivation for the common inverse/forward error scaling, that can reduce significantly the computational cost of the whole strategy. A short illustrative numerical example is attached at the end of the paper.
\end{abstract}

Keywords: Inverse problem $\cdot$ Memetic algorithm $\cdot$ Stabilized Petrov-Galerkin method

\section{Ill-Conditioned Data Inversion: State of the Art}

We will focus on Inverse Problems (IPs) which consist in finding a set of parameters $\mathcal{S}$ that minimize a misfit functional $f$ over an admissible set of parameters $\mathcal{D} \subset \mathbb{R}^{N}$, i.e.:

$$
\mathcal{D} \supset \mathcal{S}=\underset{\omega \in \mathcal{D}}{\arg \min } f\left(d_{0}, u(\omega)\right),
$$

where $u(\omega) \in U$ is a solution of the forward (direct) problem

$$
B(\omega ; u(\omega))=l
$$

corresponding to the parameter $\omega \in \mathcal{D}, d_{0} \in \mathcal{O}$ denotes the observed data and $f: \mathcal{O} \times U \rightarrow \mathbb{R}_{+}$. The forward problem operator $B: \mathcal{D} \times U \rightarrow V^{\prime}$ forms a mathematical model of the studied phenomenon, $U$ is a Hilbert space of forward solutions and $l \in V^{\prime}$ is a functional over a Hilbert space of "test functions" $V$.

\footnotetext{
The work was supported by the National Science Centre, Poland, grant No. 2017/26/M/ST1/00281.

(C) The Author(s) 2021

M. Paszynski et al. (Eds.): ICCS 2021, LNCS 12743, pp. 343-357, 2021.

https://doi.org/10.1007/978-3-030-77964-1_27
} 
If (1) has more than one solution $(\operatorname{card}(\mathcal{S})>1)$ then it becomes ill-conditioned. If $\mathcal{S}$ is not connected, then IP is called multimodal. If $\mathcal{S}$ contains an open set in $\mathbb{R}^{N}$, then we have insensitivity region (plateau) in the objective (misfit) landscape (see [11] for details). Traditionally, one can handle IP's ill conditioning by supplementing misfit with a regularization term to make it globally or locally convex (see e.g. [4]). Unfortunately, such methods can produce undesirable artifacts and lead to the loss of information regarding the modeled process. In the worst cases this method can deliver outright false solutions, forced by the regularization term.

A more sophisticated way of dealing with such IPs is to use stochastic global optimization methods which are able to identify separate basins of attraction to global minimizers (see e.g. [13]). Papers [2,3] show the application of a hierarchic evolutionary strategy HGS combined with $h p$-FEM to the identification of ambiguous Lamme coefficients in linear elasticity. The use of the same combined strategy in the inversion of logging data obtained with DC and AC probes is described in $[9,16]$. The paper [14] shows the method of misfit insensitivity regions approximation applied to MT data inversion. A hierarchic memetic strategy $h p$-HMS supplemented with an evolutionary algorithm using multiwinner selection [8] separates such areas around multiple global minimizers of the misfit.

The above-mentioned papers show an effective method of solving ill-conditioned IPs when the solution space coincides with the test space of the forward problems (i.e., $U=V$ ) and the forward operator $B$ is generally coercive ${ }^{1}$. In this paper we show how to extend the method to the class of forward problems in which $U \neq V$ and $B$ satisfies the inf-sup condition only. Such forward problems are also difficult to solve, because of the huge difference between the norms of particular components of their governing equations (e.g. diffusion-convection flow problem). Special numerical methods with stabilization such as DPG [6] have to be applied in such cases. There are few examples of inverse solutions in which DPG was applied for solving forward problem. In this number Bramwell in his dissertation [5] discusses the tomography problem of restoring the squared material slowness coefficient, using the measured displacements resulted from the point-wise harmonic stimulation on the domain surface.

\section{$2 \quad h p$-HMS as an Effective Inverse Solver}

\subsection{Evolutionary Core}

$h p$-HMS is a complex strategy that consists of a multi-phase multi-population evolutionary algorithm combined with local search, cluster detection and local approximation methods. The multi-population evolutionary core is responsible for the discovery of problem objective local minima, including more interesting global minima and these, in the inverse problem case, are solutions for (1). This global search is performed by a tree of concurrently running single-population

\footnotetext{
${ }^{1}$ This is not the case of AC and MT problems, but there we also have $U=V$.
} 
evolutionary algorithms, called demes in the sequel. The whole tree executes subsequent global steps called metaepochs. Each metaepoch in turn consists of running a fixed number of evolutionary steps in each active deme. The tree itself evolves according to the following rules:

- it starts with a single root,

- it has a fixed maximal depth,

- after a metaepoch each deme that has spotted a promising solution and is not at the deepest level can try to sprout a child deme with a population located in the neighborhood of the spotted solution.

A new deme can be sprouted only if the newfound solution is not too close to other child demes. The main idea is that the search performed in a child deme is more focused and more accurate than the one performed in the parent. The structure of the tree is then determined by the parent-child relation among demes with depth levels related to levels of search precision. The root searches the most chaotically but over the widest domain and the final solutions result from the most focused search in leaves. Any solution found in leaf demes that has a decent objective value is memorized for the further investigation. The evolution in any deme is controlled with a local stopping condition that deactivates stagnant searches. The whole strategy evolution is stopped when a global stopping condition is satisfied.

\subsection{Handling Multimodality and Insensitivity}

The sprouting condition forbids the exploration of neighborhoods of already found solutions. That forces the whole strategy to move to other areas of the computational domain and makes it possible to spot multiple different solutions. In some problems this can mean all solutions, of course when using an appropriate global stopping condition. This way, the HMS can handle multi-modal problems of a simpler type having many isolated solutions. But in some problems the difficulty level is raised higher with solutions forming plateaus, i.e., sets with nonempty interior. The evolutionary HMS core is not capable of coping with such difficulties, but the whole strategy has additional modules for this purpose. First, leaf node populations provide a hint of local minima attraction basins: among them the above-mentioned plateaus. Second, these populations are merged pairwise if they cover the same basin. Third, an evolutionary algorithm with special-type multiwinner-voting-based selection operator is run on those merged populations to enhance the coverage of the basins. Finally, on each of the basins we build a local approximation of the objective and appropriate level sets of these approximations serve as estimates of plateau boundaries.

\subsection{Computation Speedup with Dynamic Accuracy Adjustment}

It is well-known that inverse computations are very time-expensive. The main weight of these is the cost of direct problem solution. HMS inverse computations 
cannot entirely avoid this problem but in some classes of IPs we can mitigate it significantly: this is the case of solving the direct problem by means of an adaptive solver. Here we provide the idea of taking the advantage of the direct solver $h p$-adaptivity, which leads to a version of HMS called $h p$-HMS.

As it was said before, we assume that we compute the objective $\mathcal{C}(u(\omega))=$ $f\left(d_{o}, u(\omega)\right)$ with an $h p$-FEM forward problem (2) solver. In some important cases (see below) we have the following estimate

$$
\begin{aligned}
&\left.\mid \mathcal{C}\left(u_{\frac{h}{2}, p+1}(\omega)\right)-\mathcal{C}\left(u\left(\omega^{*}\right)\right)\right) \mid \leq \\
& A_{1} \operatorname{err}_{\text {rel }}(\omega)^{\alpha}+A_{2}\left\|u_{h, p}(\omega)-u(\omega)\right\|_{U}^{\beta}+A_{3}\left\|\omega-\omega^{*}\right\|_{\mathcal{D}}^{\gamma} .
\end{aligned}
$$

In formula $(3) u(\omega)$ is the solution of the direct (forward) problem $(2), u_{h, p}(\omega)$ is the $h p$-FEM approximate solution of (2) for mesh size $h$ and polynomial degree $p$, $\operatorname{err}_{r e l}(\omega)$ is a measure of the difference between approximations obtained in two subsequent $h p$-FEM steps

$$
e_{r e l}(\omega)=u_{\frac{h}{2}, p+1}(\omega)-u_{h, p}(\omega),
$$

$A_{1}, A_{2}, A_{3}>0$ are positive constants and $\alpha \geq 1, \beta \geq 1$. In the simplest case

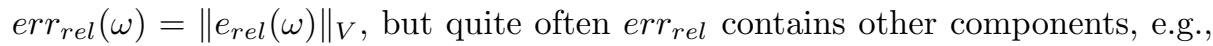
coming from the approximation of the dual problem to (2).

Here we include a proof of (3) in a quite simple but at the same time also quite general case.

Proposition 1. Assume that there exist $C_{u}>0, C_{C}>0, r \geq 1, s \geq 1$ such that

$$
\left\|u(\omega)-u\left(\omega^{\prime}\right)\right\|_{U} \leq C_{u}\left\|\omega-\omega^{\prime}\right\|_{\mathcal{D}}^{r}
$$

for every $\omega, \omega^{\prime} \in \mathcal{D}$ and that

$$
\left|\mathcal{C}(u)-\mathcal{C}\left(u^{\prime}\right)\right| \leq C_{\mathcal{C}}\left\|u-u^{\prime}\right\|^{s}
$$

for all $u$ and $u^{\prime}$. Then there exist $A_{1}>0$ and $A_{3}>0$ such that

$$
\begin{aligned}
& \left.\mid \mathcal{C}\left(u_{\frac{h}{2}, p+1}(\omega)\right)-\mathcal{C}\left(u\left(\omega^{*}\right)\right)\right) \mid \\
& \quad \leq A_{1}\left\|u_{\frac{h}{2}, p+1}(\omega)-u_{h, p}(\omega)\right\|^{s}+A_{1}\left\|u_{h, p}(\omega)-u(\omega)\right\|^{s}+A_{3}\left\|\omega-\omega^{*}\right\|^{r s} .
\end{aligned}
$$

Proof. Let us start with a simple equality

$$
u_{\frac{h}{2}, p+1}(\omega)-u\left(\omega^{*}\right)=u_{\frac{h}{2}, p+1}(\omega)-u_{h, p}(\omega)+u_{h, p}(\omega)-u(\omega)+u(\omega)-u\left(\omega^{*}\right) .
$$

Using the triangle inequality we obtain

$\left\|u_{\frac{h}{2}, p+1}(\omega)-u\left(\omega^{*}\right)\right\| \leq\left\|u_{\frac{h}{2}, p+1}(\omega)-u_{h, p}(\omega)\right\|+\left\|u_{h, p}(\omega)-u(\omega)\right\|+\left\|u(\omega)-u\left(\omega^{*}\right)\right\|$.

From (6) and (8) we have that

$$
\begin{aligned}
& \left.\mid \mathcal{C}\left(u_{\frac{h}{2}, p+1}(\omega)\right)-\mathcal{C}\left(u\left(\omega^{*}\right)\right)\right) \mid \\
& \quad \leq C_{\mathcal{C}}\left(\left\|u_{\frac{h}{2}, p+1}(\omega)-u_{h, p}(\omega)\right\|+\left\|u_{h, p}(\omega)-u(\omega)\right\|+\left\|u(\omega)-u\left(\omega^{*}\right)\right\|\right)^{s} .
\end{aligned}
$$


Then, using Jensen's inequality we obtain

$$
\begin{aligned}
& \left.\mid \mathcal{C}\left(u_{\frac{h}{2}, p+1}(\omega)\right)-\mathcal{C}\left(u\left(\omega^{*}\right)\right)\right) \mid \\
& \leq 3^{s-1} C_{\mathcal{C}}\left(\left\|u_{\frac{h}{2}, p+1}(\omega)-u_{h, p}(\omega)\right\|^{s}+\left\|u_{h, p}(\omega)-u(\omega)\right\|^{s}+\left\|u(\omega)-u\left(\omega^{*}\right)\right\|^{s}\right) .
\end{aligned}
$$

Now, the application of (5) completes the proof with

$$
A_{1}=3^{s-1} C_{\mathcal{C}}, \quad A_{3}=3^{s-1} C_{\mathcal{C}} C_{u} .
$$

Constants $C_{u}$ and $r$ are related to properties of forward equation (2). Here, we show their derivation in an important special case.

Proposition 2. Assume that in the forward problem (2) $U=V$ and that the forward operator $B$ has the following properties.

1. For every $\omega \in \mathcal{D} B(\omega ; u)$ is linear and continuous with respect to $u$.

2. $B(\omega ; u)$ is uniformly coercive with respect to $u$, i.e. there exists $C_{b}>0$ independent on $\omega$ such that

$$
|\langle B(\omega ; u), u\rangle| \geq C_{b}\|u\|_{U}^{2}
$$

3. $B(\omega ; u)$ is uniformly Lipschitz-continuous with respect to $\omega$, i.e. there exist $C_{B}>0$ independent on $u$ such that

$$
\left\|B(\omega ; u)-B\left(\omega^{\prime} ; u\right)\right\|_{U^{\prime}} \leq C_{B}\|u\|_{U}\left\|\omega-\omega^{\prime}\right\|_{\mathcal{D}}
$$

Then (5) holds with $r=1$ and

$$
C_{u}=\frac{C_{B}}{C_{b}^{2}}\|l\|_{U^{\prime}} .
$$

Proof. In the sequel we shall use the following notation

$$
B(\omega): U \ni u \longmapsto B(\omega ; u) \in U^{\prime} .
$$

Then, we can state that

$$
u(\omega)=B(\omega)^{-1}(l) .
$$

We have the following sequence of equalities.

$$
\begin{aligned}
u(\omega)-u\left(\omega^{\prime}\right) & =B(\omega)^{-1}(l)-B\left(\omega^{\prime}\right)^{-1}(l) \\
& =\left[B(\omega)^{-1} \circ\left(B\left(\omega^{\prime}\right)-B(\omega)\right) \circ B\left(\omega^{\prime}\right)^{-1}\right](l) .
\end{aligned}
$$

Therefore, using operator-norm definition we obtain

$$
\left\|u(\omega)-u\left(\omega^{\prime}\right)\right\| \leq\left\|B(\omega)^{-1}\right\| \cdot\left\|B\left(\omega^{\prime}\right)-B(\omega)\right\| \cdot\left\|B\left(\omega^{\prime}\right)^{-1}\right\| \cdot\|l\| .
$$


Thanks to Assumption 2 we have

$$
C_{b}\|u\|^{2} \leq|\langle B(\omega ; u), u\rangle| \leq\|B(\omega ; u)\|\|u\| .
$$

Therefore, again from the definition of the operator norm, it follows that

$$
\|B(\omega ; u(\omega))\| \geq C_{b}\|u(\omega)\| .
$$

Using (9) we can rewrite the above in the following way

$$
\left\|B(\omega)^{-1}(L)\right\| \leq \frac{1}{C_{b}}\|l\|
$$

Hence, for every $\omega \in \mathcal{D}$ we have

$$
\left\|B(\omega)^{-1}\right\| \leq \frac{1}{C_{b}}
$$

Assumption 3 can be rewritten in the following way

$$
\left\|B(\omega)-B\left(\omega^{\prime}\right)\right\| \leq C_{B}\left\|\omega-\omega^{\prime}\right\| .
$$

Hence, using the above inequalities along with (10) we obtain

$$
\left\|u(\omega)-u\left(\omega^{\prime}\right)\right\| \leq \frac{1}{C_{b}} \cdot C_{B} \cdot\left\|\omega-\omega^{\prime}\right\| \cdot \frac{1}{C_{b}}\|l\|,
$$

which concludes the proof.

The first right-hand-side component of (3) contains a power of the error loss $\operatorname{err}_{r e l}(\omega)$ in a single $h p$-FEM step. The last component is a power of the error of the inverse problem solution, that is related to the assumed search range on a given level of the HMS tree. When using an $h p$-adaptive direct solver we can trade the precision of the computations for the savings in time and reversely we can spend more time to obtain more accurate solution. The main idea of the $h p$-HMS is then to dynamically adjust the accuracy of the misfit evaluation to both a particular value of $\omega$ and the (inevitable) inverse problem solution error characterizing a given HMS tree level. The adjustment is realized by keeping the balance between the first and the last right-hand term in (3): the middle term can be neglected due to the high rate of the $h p$-FEM convergence. Therefore, if $\delta_{j}$ is an assumed precision of the inverse problem solution (i.e., an assumed level of the inverse error) at level $j$ we perform the $h p$-adaptation of the FEM solution of the forward problem until $\operatorname{err}_{r e l}(\omega)$ drops below $\operatorname{Ratio}(j) \delta_{j}{ }^{\frac{1}{\alpha}}$, where $\operatorname{Ratio}(j)$ is a parameter of the strategy related to the constant $\left(A_{3}\left(A_{1}\right)^{-1}\right)^{\frac{1}{\alpha}}$.

Remark 1. Such a dynamical accuracy adjustment can result in a notable reduction of the computational cost of $h p$-HMS: we refer the reader to papers $[2,15]$ for details. 


\section{Stabilizing Forward Petrov-Galerkin Based Solver by Using Demkowicz Operator}

\subsection{Sample Exact Forward Problem}

Let us study the following variational forward problem:

$$
\left\{\begin{array}{c}
\text { Find } u \in U ; \\
b(u, v)=l(v) \forall v \in V
\end{array}\right\} \Leftrightarrow\left\{\begin{array}{c}
B u=l, \quad B: U \rightarrow V^{\prime} \\
\langle B u, v\rangle_{V^{\prime} \times V}=b(u, v) \forall v \in V
\end{array}\right\}
$$

where $U, V$ are two real Hilbert spaces, $b: U \times V \rightarrow \mathbb{R}$ is a bilinear (sesquilinear), continuous form, $l: V \rightarrow \mathbb{R}$ continuous linear functional so $|b(u, v)| \leq$ $M\|u\|_{U}\|v\|_{V},|l(v)| \leq\|l\|_{V^{\prime}}\|u\|_{U}$ where $M$ stands for the norm of the form $b$.

If $b$ satisfies the following inf-sup condition equivalent to the condition, that $B$ is bounded below:

$$
\exists \gamma>0 ; \forall u \in U \sup _{0 \neq v \in V}\left\{\frac{|b(u, v)|}{\|v\|_{V}} \geq \gamma\|u\|_{U}\right\}
$$

and $l$ satisfies the compatibility condition

$$
l(v)=0 \forall v \in V_{0}=\operatorname{ker}\left(B^{\prime}\right)=\{v \in V ; b(u, v)=0 \forall u \in U\},
$$

or the stronger one $\operatorname{ker}\left(B^{\prime}\right)=\{v \in V ; b(u, v)=0 \forall u \in U\}=\{0\}$, then (11) has a unique solution that satisfies the stability estimate $\|u\|_{U} \leq \frac{1}{\gamma}\|l\|_{V^{\prime}}$, where $B^{\prime}:\left(V^{\prime}\right)^{\prime}=V \ni v \rightarrow\left\langle\cdot, B^{\prime} v\right\rangle_{V^{\prime} \times V}=b(\cdot, v) \in U^{\prime}$, because $V$ is reflexive as a Hilbert space. Moreover $B$ is the isomorphism (see e.g. Babuška [1] and references inside).

\subsection{Petrov-Galerkin Method}

By choosing $U_{h} \subset U, V_{h} \subset V$; $\operatorname{dim}\left(U_{h}\right)=\operatorname{dim}\left(V_{h}\right)<+\infty$ the problem approximate to (11) can be obtained:

$$
\text { Find } u_{h} \in U_{h} ; \quad b\left(u_{h}, v\right)=l(v) \forall v \in V_{h}
$$

If (14) satisfies the discrete inf-sup condition:

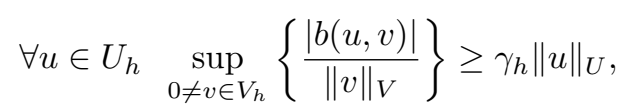

then it has the unique solution $u_{h}$ which satisfies the discrete stability condition $\left\|u_{h}\right\|_{U} \leq \frac{1}{\gamma_{h}}\|l\|_{V^{\prime}}$, moreover if $u$ is the solution to (11) then

$$
\left\|u-u_{h}\right\|_{U} \leq \frac{M}{\gamma_{h}} \inf _{w_{h} \in U_{h}}\left\{\left\|u-w_{h}\right\|_{U}\right\}
$$


If the stability constants $\gamma_{h}$ have a positive lover bound $\inf _{h}\left\{\gamma_{h}\right\}=\gamma_{0}>0$, then the Petrov-Galerkin method error converges with the same rate as the best approximation error, because:

$$
\left\|u-u_{h}\right\|_{U} \leq \frac{M}{\gamma_{0}} \inf _{w_{h} \in U_{h}}\left\{\left\|u-w_{h}\right\|_{U}\right\} .
$$

Unfortunately, the continuous inf-sup condition does not imply uniform discrete inf-sup condition, so (17) does not hold in general for the arbitrary spaces $U_{h} \subset U, V_{h} \subset V$ (see Babuška [1]).

\subsection{Demkowicz Operator}

The way to overcome this obstacle was introduced by Demkowicz and his collaborators $[6,7]$.

Let $\mathcal{H}$ be the set of all finite dimensional subspaces of $U$ and $\mathcal{B}$ the set of all finite dimensional subspaces of $V$. We are looking for any mapping $\Im: \mathcal{H} \rightarrow \mathcal{B}$ so that (14) is symmetric and uniformly stable $((15)$ is satisfied with a uniform constant) for each pair $\left(U_{h}, V_{h}=\Im\left(U_{h}\right)\right), U_{h} \in \mathcal{H}$.

Let $R_{V}: V \rightarrow V^{\prime}$ be the Riesz isometry, then $\forall u \in U\|B u\|_{V^{\prime}}=\left\|R_{V}^{-1} B u\right\|_{V}$. We define now the linear Demkowicz operator

$$
T=R_{V}^{-1} B: U \rightarrow V
$$

$T$ is an isomorphism as a composition of isomorphisms, moreover

$$
\forall u \in U \quad\|B u\|_{V^{\prime}}=\left\|R_{V}^{-1} B u\right\|_{V}=\|T u\|_{V} .
$$

It can be proved, that $\Im\left(U_{h}\right) \equiv T U_{h}$ satisfies our needs.

First, setting $V_{h}=T U_{h}$ we obtain $\operatorname{dim}\left(V_{h}\right)=\operatorname{dim}\left(U_{h}\right)=n$, because $T$ is the isomorphism and next:

$$
\forall 0 \neq u \in U\|B u\|_{V^{\prime}}=\sup _{0 \neq v \in V}\left\{\frac{|b(u, v)|}{\|v\|_{V}}\right\}=\frac{|b(u, T u)|}{\|T u\|_{V}}
$$

Let us introduce the energy norm $\|\cdot\|_{E}$ on the space $U$ so that

$$
\|u\|_{E}=\|T u\|_{V}=\left\|R_{V}^{-1} B u\right\|_{V}=\|B u\|_{V^{\prime}}=\sup _{0 \neq v \in V}\left\{\frac{|b(u, v)|}{\|v\|_{V}}\right\} .
$$

Both norms $\|\cdot\|_{E}$ and $\|\cdot\|_{U}$ are equivalent, because

$$
\gamma\|u\|_{U} \leq \sup _{0 \neq v \in V}\left\{\frac{|b(u, v)|}{\|v\|_{V}}\right\}=\|u\|_{E}=\|B u\|_{V^{\prime}} \leq M\|u\|_{U},
$$

and it is easy to prove:

Lemma 1. If $V_{h}=T U_{h}$, then the inf-sup constant in (15) equals $\gamma_{h}=1$ with respect to the norm $\|\cdot\|_{E}$ independently on the selection of the space $U_{h} \subset U$. 


\subsection{The Abstract Stabilized Forward Problem}

Now we are ready to introduce the following form of the stabilized PetrovGalerkin forward problem:

$$
\text { Find } u_{h} \in U_{h} ; d\left(u_{h}, w\right)=r(w) \forall w \in U_{h},
$$

where $d: U \times U \ni u, w \rightarrow d(u, w)=b(u, T w) \in \mathbb{R}$ and $r: U \ni w \rightarrow r(w)=$ $l(T w) \in \mathbb{R}$.

Remark 2. The problems (14) and (23) are equivalent, if $V_{h}=T U_{h}$.

\section{Theorem 1.}

1. $d$ is symmetric on $U \times U$

2. $d(u, u)=\|u\|_{E}^{2} \forall u \in U$

3. $|d(u, w)| \leq\|u\|_{E}\|w\|_{E} \forall u, w \in U$

4. $|r(w)| \leq\|l\|_{V^{\prime}}\|w\|_{E} \forall w \in U$

Theorem 2. The problem (23) has the unique solution $u_{h} \in U_{h}$ so that $\left\|u_{h}\right\|_{E} \leq$ $\|l\|_{V^{\prime}}$. Moreover $\left\|u-u_{h}\right\|_{E}=\inf _{w \in U_{h}}\left\{\|u-w\|_{E}\right\}$, where $u \in U$ is the solution to (11).

Proof of Theorem 1: $d(u, w)=b(u, T w)=\langle B u, T w\rangle_{V^{\prime} \times V}=\left(R_{V}^{-1} B u, T w\right)_{V}=$ $(T u, T w)_{V}$, so $d$ satisfies 1 . Next $d(u, u)=(T u, T u)_{V}=\|T u\|_{V}^{2}=\|u\|_{E}^{2}$ proves 2. and $|d(u, w)|=\left|(T u, T w)_{V}\right| \leq\|T u\|_{V}\|T w\|_{V}=\|u\|_{E}\|w\|_{E}$ proves 3. Finally, $|r(w)| \leq\|l\|_{V^{\prime}}\|T w\|_{V}=\|l\|_{V^{\prime}}\|w\|_{E}$ proves 4 .

Proof of Theorem 2: The form $d$ is a scalar product on $U$ inducing the energy norm $\|\cdot\|_{E}$. Moreover $d$ and $r$ preserve their conditions while restricting to the subspace $U_{h}$, so the first thesis follows from Riesz theorem for this subspace. The second thesis immediately follows from the Céa lemma which implies $\left\|u-u_{h}\right\|_{E} \leq$ $\inf _{w \in U_{h}}\left\{\|u-w\|_{E}\right\}$, but $\left\|u-u_{h}\right\|_{E}$ must be less or equal to $\inf _{w \in U_{h}}\left\{\|u-w\|_{E}\right\}$ because both $u_{h}, w \in U_{h}$.

Remark 3. All results of Theorems 1 and 2 do not depend on the selection of the approximation subspace $U_{h} \subset U$.

Remark 4. Both, the energy norm $\|\cdot\|_{E}$ and Demkowicz isomorphism $T$ depend on the variational problem to be solved. Moreover, the conditions of each problem (23) determined by Theorems 1, 2 can be expressed using the norm $\|\cdot\|_{U}$ and constants $M, \gamma$ characterizing the exact problem (11). In particular

$$
\left\|u-u_{h}\right\|_{U} \leq \frac{M}{\gamma} \inf _{w_{h} \in U_{h}}\left\{\left\|u-w_{h}\right\|_{U}\right\}
$$

moreover:

$$
\begin{aligned}
& d(u, u) \geq \gamma^{2}\|u\|_{U}^{2},|d(u, w)| \leq M^{2}\|u\|_{U}\|w\|_{U} \\
& |r(w)| \leq\|l\|_{V^{\prime}} M\|w\|_{U} \quad \forall u, w \in U
\end{aligned}
$$




\section{Including Demkowicz Operator in the hp-HMS Structure}

Let us assume, that the HMS objective $\mathcal{C}(u(\omega))$ fits the misfit of the inverse problem (1) and satisfies the inequality:

$$
\begin{aligned}
& \mathcal{C}(u(\omega))=f\left(d_{o}, u(\omega)\right) \quad \forall \omega \in \mathcal{D}, \\
& \left|\mathcal{C}(u)-\mathcal{C}\left(u^{\prime}\right)\right| \leq C_{C}\left\|u-u^{\prime}\right\|_{U}^{s} \quad \forall u, u^{\prime} \in U,
\end{aligned}
$$

where $s, C_{C}$ are some positive constants and $u(\omega) \in U$ is the solution to the following forward problem:

$$
\begin{gathered}
\left\{\begin{array}{c}
\text { Find } u(\omega) \in U ; \\
b(\omega ; u(\omega), v)=l(v) \forall v \in V
\end{array}\right\} \\
\mathbb{\Downarrow} \\
\left\{\begin{array}{c}
B(\omega ; u(\omega))=l, \quad B(\omega ; \cdot): U \rightarrow V^{\prime} ; \\
\left\langle B(\omega ; u, v\rangle_{V^{\prime} \times V}=b(\omega ; u, v) \forall u \in U, \forall v \in V\right.
\end{array}\right\}
\end{gathered}
$$

where $U, V$ are two real Hilbert spaces, $b(\omega ; \cdot, \cdot): U \times V \rightarrow \mathbb{R}$ is a bilinear (sesquilinear), continuous form, $l: V \rightarrow \mathbb{R}$ continuous linear functional so $|b(\omega ; u, v)| \leq M_{\omega}\|u\|_{U}\|v\|_{V},|l(v)| \leq\|l\|_{V^{\prime}}\|u\|_{U}$ where $M_{\omega}$ stands for the norm of the form $b(\omega ; \cdot, \cdot)$.

If $b(\omega ; \cdot, \cdot)$ satisfies the following inf-sup condition:

$$
\exists \gamma_{\omega}>0 ; \forall u \in U \sup _{0 \neq v \in V}\left\{\frac{|b(\omega ; u, v)|}{\|v\|_{V}} \geq \gamma_{\omega}\|u\|_{U}\right\}
$$

and $l$ satisfies the compatibility condition

$$
l(v)=0 \forall v \in V_{0}=\operatorname{ker}\left(B^{\prime}(\omega, \cdot)\right)=\{v \in V ; b(\omega ; u, v)=0 \forall u \in U\},
$$

or the stronger one $\operatorname{ker}\left(B^{\prime}(\omega ; \cdot)\right)=\{v \in V ; b(\omega ; u, v)=0 \forall u \in U\}=\{0\}$, then (27) has a unique solution that satisfies the stability estimate $\|u(\omega)\|_{U} \leq$ $\frac{1}{\gamma_{\omega}}\|l\|_{V^{\prime}}$, where $B^{\prime}(\omega ; \cdot):\left(V^{\prime}\right)^{\prime}=V \ni v \rightarrow\left\langle\cdot, B^{\prime}(\omega ; \cdot) v\right\rangle_{V^{\prime} \times V}=b(\omega ; \cdot, v) \in U^{\prime}$, because $V$ is reflexive as a Hilbert space. Moreover $B(\omega ; \cdot)$ is the isomorphism (see e.g. Babuška [1] and references inside). Next we assume that

$$
\exists \gamma, M ;+\infty>M=\sup _{\omega \in \mathcal{D}}\left\{M_{\omega}\right\}, 0<\gamma=\inf _{\omega \in \mathcal{D}}\left\{\gamma_{\omega}\right\}
$$

Moreover, we assume that $\mathcal{D}$ is bounded and that

$$
\exists C_{F}>0 ;\left\|B(\omega ; u)-B\left(\omega^{\prime} ; u\right)\right\|_{V^{\prime}} \leq C_{F}\|u\|_{U}\left\|\omega-\omega^{\prime}\right\|_{\mathcal{D}} \forall u \in U, \forall \omega, \omega^{\prime} \in \mathcal{D} .
$$

In the sequel we shall use the following notation

$$
\begin{aligned}
& T(\omega ; \cdot)=R_{V}^{-1} B(\omega ; \cdot): U \rightarrow V \\
& d(\omega ; u, v)=b(\omega ; u, T(\omega ; v)), D(\omega ; u): U \rightarrow U^{\prime} \\
& \langle D(\omega ; u), v\rangle_{U^{\prime} \times U}=d(\omega ; u, v)=b(\omega ; u, T(\omega ; v)) \quad \forall u, v \in U .
\end{aligned}
$$


Proposition 3. The family of operators $\{D(\omega ; \cdot)\} . \omega \in \mathcal{D}$ satisfies the assumptions of Proposition 2.

Proof. Because $\langle D(\omega ; u), v\rangle_{U^{\prime} \times U}=b(\omega ; u, T(\omega ; v))$ than $D(\omega ; \cdot)$ is linear and continuous for every $\omega \in \mathcal{D}$, which satisfies the assumption 1 .

Following Remark 4 and the assumption (30) we have

$$
\langle D(\omega ; u), u\rangle_{U^{\prime} \times U}=d(\omega ; u, u) \geq \gamma_{\omega}^{2}\|u\|^{2} \geq \gamma^{2}\|u\|^{2}
$$

which proves the uniform coercivity of $D(\omega ; \cdot)$ postulated in assumption 2 .

Let us evaluate for arbitrary $\omega, \omega^{\prime} \in \mathcal{D}, u, v \in U$ and using (30), (31)

$$
\begin{aligned}
& \left|d(\omega ; u, v)-d\left(\omega^{\prime} ; u, v\right)\right|=\left|b(\omega ; u, T(\omega, v))-b\left(\omega^{\prime} ; u, T\left(\omega^{\prime} ; v\right)\right)\right| \\
& \leq\left|b(\omega ; u, T(\omega, v))-b\left(\omega^{\prime} ; u, T(\omega ; v)\right)\right|+\left|b\left(\omega^{\prime} ; u, T(\omega ; v)\right)-b\left(\omega^{\prime} ; u, T\left(\omega^{\prime} ; v\right)\right)\right| \\
& \leq\left\|B(\omega ; u)-B\left(\omega^{\prime} ; u\right)\right\|_{V^{\prime}}\|T(\omega ; v)\|_{U}+\left|b\left(\omega^{\prime} ; u, T(\omega ; v)-T\left(\omega^{\prime} ; v\right)\right)\right| \\
& \leq C_{F}\left\|\omega-\omega^{\prime}\right\|_{\mathcal{D}}\|u\|_{U}\|T(\omega ; v)\|_{U}+\left\|B\left(\omega^{\prime}\right)\right\|\|u\|_{U}\left\|T(\omega ; v)-T\left(\omega^{\prime} ; v\right)\right\|_{V} \\
& \leq C_{F}\|u\|_{U}\left\|\omega-\omega^{\prime}\right\|_{\mathcal{D}}\|B(\omega ; v)\|_{V^{\prime}}+\left\|B\left(\omega^{\prime}\right)\right\|\|u\|_{U}\left\|B(\omega ; v)-B\left(\omega^{\prime} ; v\right)\right\|_{V^{\prime}} \\
& \leq C_{F}\|u\|_{U}\left\|\omega-\omega^{\prime}\right\|_{\mathcal{D}}\|B(\omega)\|\|v\|_{V}+\left\|B\left(\omega^{\prime}\right)\right\|\|u\|_{U}\left\|\cdot C_{F}\right\| \omega-\omega^{\prime}\left\|_{\mathcal{D}}\right\| v \|_{V} \\
& \leq C_{F}\left(\|B(\omega)\|+\left\|B\left(\omega^{\prime}\right)\right\|\right)\left\|\omega-\omega^{\prime}\right\|_{\mathcal{D}}\|u\|_{U}\|v\|_{V}
\end{aligned}
$$

(31) can be rewritten as

$$
\left\|B(\omega)-B\left(\omega^{\prime}\right)\right\| \leq C_{F}\left\|\omega-\omega^{\prime}\right\|_{\mathcal{D}}
$$

therefore, since $\mathcal{D}$ is bounded, we have that

$$
M_{B}=\sup _{\omega \in \mathcal{D}}\|B(\omega)\|<+\infty
$$

Hence, we obtain

$$
\left|d(\omega ; u, v)-d\left(\omega^{\prime} ; u, v\right)\right| \leq 2 C_{F} M_{B}\left\|\omega-\omega^{\prime}\right\|_{\mathcal{D}}\|u\|_{U}\|v\|_{V}
$$

In other words

$$
\left\|D(\omega ; u)-D\left(\omega^{\prime} ; u\right)\right\|_{V^{\prime}} \leq 2 C_{F} M_{B}\left\|\omega-\omega^{\prime}\right\|_{\mathcal{D}}\|u\|_{U}
$$

which is exactly assumption 3 .

Finally, taking into account the assumed inequality (26), all assumptions of the Proposition 1 hold, so the evaluation of the misfit relative error (7) is valid with the following constants $A_{1}=3^{s-1} C_{C}, A_{3}=A_{1} C_{u}=\frac{3^{s-1} C_{C} C_{B}^{2}}{C_{b}^{2}}\|l\|_{U^{\prime}}$.

Proposition 4. All the above considerations authorize the conclusion that the common misfit error scaling described in Sect. 2.3 might be applied in twin adaptive solution of the inverse problem (1) where the forward problems (27) are stabilized by Demkowicz operator, using the recommended value of Ratio $(j)$, i.e. $\left(A_{3}\left(A_{1}\right)^{-1}\right)^{\frac{1}{\alpha}}$.

Remark 5. The above Proposition allows us to apply the dynamical accuracy adjustment technique to problems demanding stabilization as well. An important consequence is that also in this case we can expect computational cost savings similar to those mentioned in Remark 1. 


\section{$5 \quad$ Numerical Example}

In this section we shall describe a simple illustrative computational example. Our intention is to show a hint for a broader class of problems that can be attacked using stabilized FEM coupled with the accuracy-adjusting HMS. However, the problem itself is forward-only, hence it does not use any inverse solver.

Let us consider a simple advection-dominated diffusion-advection equation on a one-dimensional domain $\Omega=(0,1)$ :

$$
-\varepsilon u^{\prime \prime}+u^{\prime}=1, \quad \varepsilon \ll 1
$$

with zero Dirichlet boundary conditions. The exact solution

$$
u(x)=x-\frac{e^{x / \varepsilon}-1}{e^{1 / \varepsilon}-1}
$$

exhibits a sharp boundary layer near $x=1$, which necessitates stabilization. As a starting point we use the standard weak formulation with $U=V=H_{0}^{1}(\Omega)$ : Find $u \in H_{0}^{1}(\Omega)$ such that

$$
\varepsilon\left(u^{\prime}, v^{\prime}\right)+\left(u^{\prime}, v\right)=(1, v) \quad \forall v \in H_{0}^{1}(\Omega)
$$

To discretize the above continuous formulation, we employ the ,practical" DPG method described in [10, Definition 31]. Having chosen the discrete trial space $U_{h}$ and a discrete test space $V^{s}$ satisfying $\operatorname{dim} U_{h}<\operatorname{dim} V^{s}$, we proceed to solve the following equivalent discrete mixed problem [10, Theorem 39]: Find $u_{h} \in U_{h}$ and $\psi \in V^{s}$ such that

$$
\begin{aligned}
\left(\psi^{\prime}, v^{\prime}\right)+\varepsilon\left(u^{\prime}, v^{\prime}\right)+\left(u^{\prime}, v\right) & =(1, v) & & \forall v \in V^{s} \\
\varepsilon\left(w^{\prime}, \psi^{\prime}\right)+\left(w^{\prime}, \psi\right) & =0 & & \forall w \in U_{h}
\end{aligned}
$$

In our numerical example we use uniform mesh with 20 elements. As the trial space $U_{h}$ we use quadratic B-splines space with $C^{1}$ global continuity, and as the discrete test space $V^{s}$ - cubic B-splines with $C^{0}$ global continuity. Figure 1 presents the exact solution (5) for $\varepsilon=1 / 125$, and approximate solutions using the described stabilization technique and the standard Galerkin method with trial and test spaces equal to $U_{h}$. In the vicinity of the boundary layer the Galerkin solution oscillates heavily, while the stabilized solution remains close to the exact solution. 


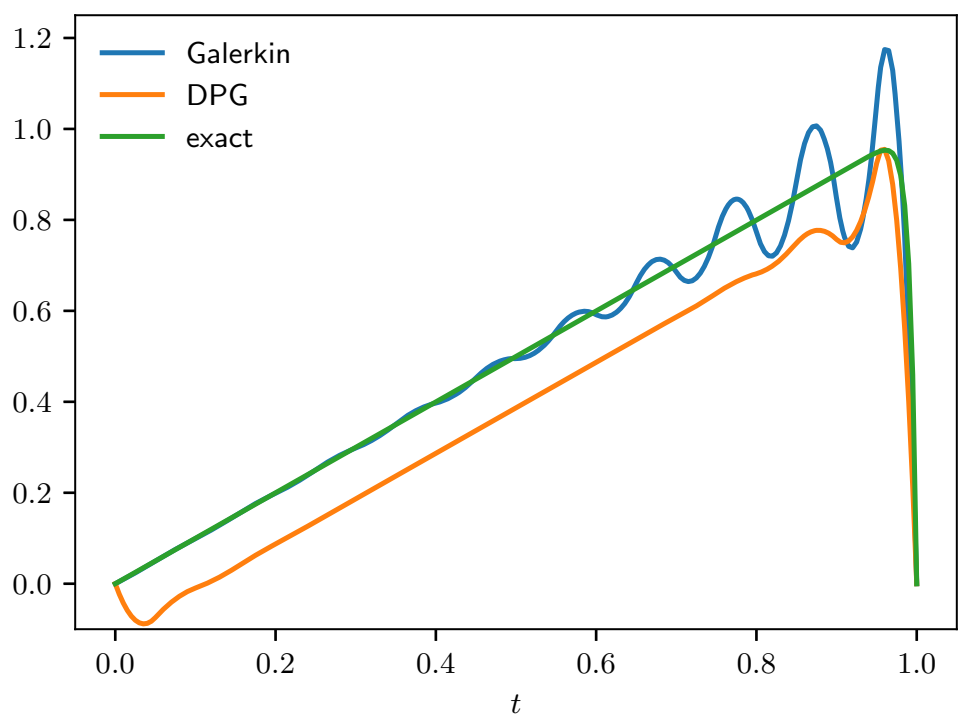

Fig. 1. Exact and approximate solutions of problem (35)

\section{Conclusions}

The paper refers to the effective stochastic strategy $h p$-HMS of solving ill conditioned parametric inverse problems. The core of this strategy is a dynamic, common inverse/forward error scaling based on the formula (3). Our earlier papers $[2,3,8,9,14,16]$ verifies this formula and the $h p$-HMS parameters setting for strongly motivated engineering problems: identification of Lamé coefficients in linear elasticity, inversion of logging data obtained with DC and AC probes and MT data inversion by underground resources investigations. For such cases the associated forward problem (2) have uniformly coercive and Lipschitz continuous operators and can be solved by the Galerkin method with the same test and trial spaces.

Considerations included in Sects. 2-4 allow to extend the above results to more difficult forward problems solved by means of the Petrov-Galerkin method, that needs to be stabilized by the Demkowicz operator. In particular, Proposition 4 specifies new constant values in (3) for the stabilized case and makes it possible to apply the same algorithmic solutions as in non-stabilized cases. The stabilization of the forward problem using the Demkowicz operator can be combined with the standard $h p$-adaptivity [12], making it appropriate for the use in double-adaptive inverse solvers. Most of the other observations concerning $h p$-HMS behavior also remain valid.

Our future work shall involve the application of the presented methods in the solution of real-world inverse problems. 


\section{References}

1. Babuška, I.: Error-bounds for finite element method. Numerische Mathematic 16, 322-333 (1971)

2. Barabasz, B., Gajda-Zagórska, E., Migórski, S., Paszyński, M., Schaefer, R., Smołka, M.: A hybrid algorithm for solving inverse problems in elasticity. Int. J. Appl. Math. Comput. Sci. 24(4), 865-886 (2014)

3. Barabasz, B., Migórski, S., Schaefer, R., Paszyński, M.: Multi-deme, twin adaptive strategy hp-HGS. Inverse Probl. Sci. Eng. 19(1), 3-16 (2011)

4. Beilina, L., Klibanov, M.V.: Approximate Global Convergence and Adaptivity for Coefficient Inverse Problems. Springer, Boston (2012). https://doi.org/10.1007/ 978-1-4419-7805-9

5. Bramwell, J.: A Discontinuous Petrov-Galerkin Method for Seismic Tomography Problems. Ph.D. thesis, The University of Texas at Austin, Austin, USA (2013)

6. Demkowicz, L., Gopalakrishnan, J.: A class of discontinuous Petrov-Galerkin method. Part I: The transport equation. Comput. Methods Appl. Mech. Eng. 199, 1558-1572 (2010). https://doi.org/10.1016/j.cma.2010.01.003

7. Demkowicz, L., Gopalakrishnan, J.: A class of discontinuous Petrov-Galerkin method. Part II: Optimal test functions. Numer. Methods Partial Differ. Equ. 27, 70-105 (2011). https://doi.org/10.1002/num.20640

8. Faliszewski, P., Sawicki, J., Schaefer, R., Smołka, M.: Multiwinner voting in genetic algorithms. IEEE Intell. Syst. 32(1), 40-48 (2017). https://doi.org/10.1109/MIS. 2017.5

9. Gajda-Zagórska, E., Schaefer, R., Smołka, M., Paszyński, M., Pardo, D.: A hybrid method for inversion of 3D DC logging measurements. Natural Comput. 14(3), 355-374 (2014). https://doi.org/10.1007/s11047-014-9440-y

10. Gopalakrishnan, J.: Five lectures on DPG methods. Master's thesis, Portland State University (2013)

11. Łoś, M., Smołka, M., Schaefer, R., Sawicki, J.: Misfit landforms imposed by illconditioned inverse parametric problems. Comput. Sci. 19 (2018). https://doi. org/10.7494/csci.2018.19.2.2781

12. Petrides, S., Demkowicz, L.: An adaptive DPG method for high frequency timeharmonic wave propagation problems. report 16-20. Technical report. The Institute for Computational Engineering and Sciences, The University of Texas at Austin (2016)

13. Preuss, M.: Multimodal Optimization by Means of Evolutionary Algorithms. Natural Computing, Springer, Cham (2015). https://doi.org/10.1007/978-3-31907407-8

14. Sawicki, J., Łoś, M., Smołka, M., Schaefer, R., Álvarez-Aramberri, J.: Approximating landscape insensitivity regions in solving ill-conditioned inverse problems. Memetic Comput. 10(3), 279-289 (2018). https://doi.org/10.1007/s12293018-0258-5

15. Schaefer, R., Kołodziej, J.: Genetic search reinforced by the population hierarchy. In: Foundations of Genetic Algorithms, vol. 7, pp. 383-399. Morgan Kaufman (2003)

16. Smołka, M., Gajda-Zagórska, E., Schaefer, R., Paszyński, M., Pardo, D.: A hybrid method for inversion of 3D AC logging measurements. Appl. Soft Comput. 36, 422-456 (2015) 
Open Access This chapter is licensed under the terms of the Creative Commons Attribution 4.0 International License (http://creativecommons.org/licenses/by/4.0/), which permits use, sharing, adaptation, distribution and reproduction in any medium or format, as long as you give appropriate credit to the original author(s) and the source, provide a link to the Creative Commons license and indicate if changes were made.

The images or other third party material in this chapter are included in the chapter's Creative Commons license, unless indicated otherwise in a credit line to the material. If material is not included in the chapter's Creative Commons license and your intended use is not permitted by statutory regulation or exceeds the permitted use, you will need to obtain permission directly from the copyright holder. 